UCRL-JC-129499

PREPRINT

\title{
Theoretical Aspects of the Use of Pulsed Reflectometry in a Spheromak Plasma
}

\author{
Bruce I. Cohen \\ E. Bickford Hooper \\ M.C. Spang \\ C.W. Domier
}

This paper was prepared for submittal to

12th Topical Conference on High Temperature Plasma Diagnostics

Princeton, NJ

June 7-11, 1998

June 11, 1998

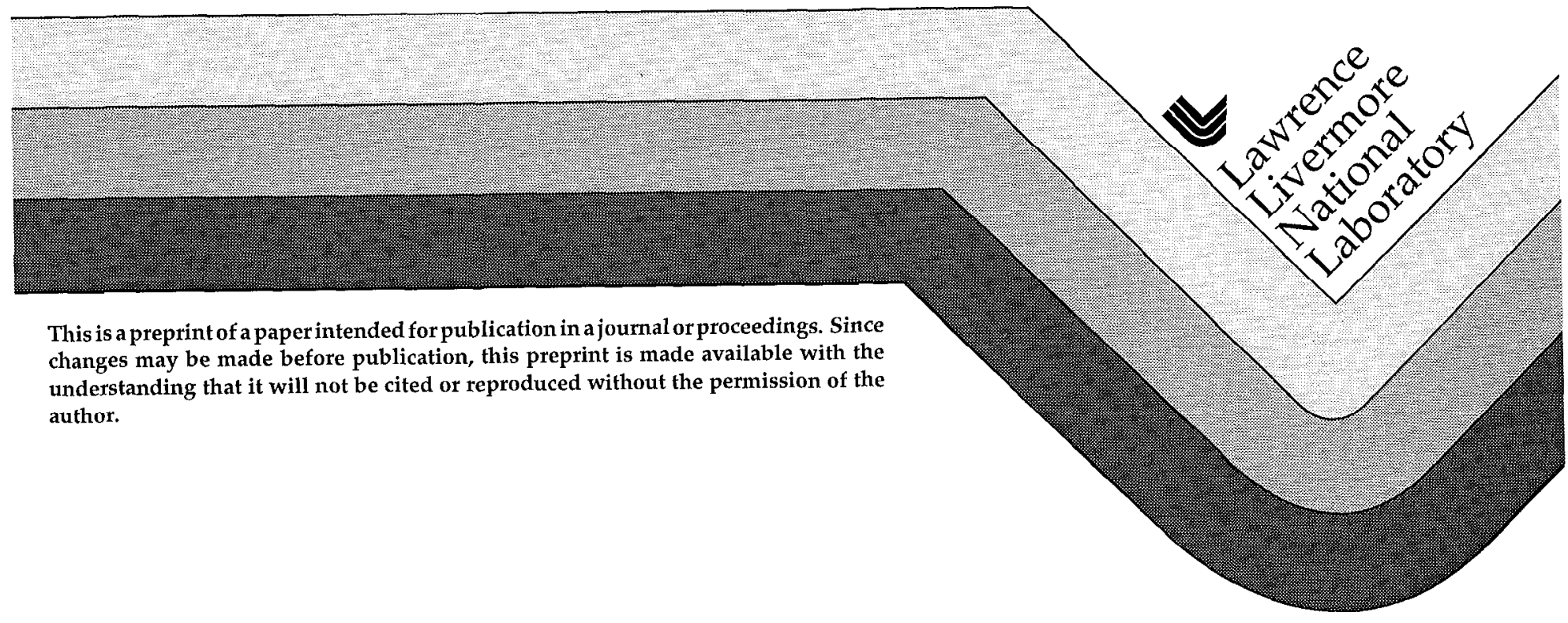


DISCLAIMER

This document was prepared as an account of work sponsored by an agency of the United States Government Neither the United States Government nor the University of California nor any of their employees, makes any warranty, express or implicd, or assumes any legal liability or responsibility for the accuracy, completeness, or usefulness of any information, apparatus, product, or process disclosed, or represents that its use would not infringe privately owned rights Reference herein to any specific commercial product, process, or service by trade name, trademark, manufacturer, or otherwise, does not necessarily constitute or imply its endorsement, recommendation, or favoring by the United States Government or the University of California. The views and opinions of authors expressed herein do not necessarily state or reflect those of the United States Government or the Iniversity of California, and shall not be used for advertising or product endorsement purposes. 


\title{
Theoretical Aspects of the Use of Pulsed Reflectometry in a Spheromak Plasma
}

\author{
Bruce I. Cohen and E. Bickford Hooper \\ Lawrence Livermore National Laboratory, University of California, \\ Livermore, California 94551 \\ M. C. Spang \\ Department of Nuclear Engineering, University of California \\ Berkeley, California 94720 \\ and C. W. Domier \\ Department of Applied Science, University of California at Davis \\ Davis, California 95616
}

\begin{abstract}
Pulsed reflectometry using both ordinary $(\mathrm{O})$ and extraordinary $(\mathrm{X})$ modes has the potential of providing time and space-resolved measurements of the electron density, the magnitude of the magnetic field, and the magnetic shear as a function of radius. Such a diagnostic also yields the current profile from the curl of the magnetic field. This research addresses theoretical issues associated with the use of reflectometry in the SSPX spheromak experiment at the Lawrence Livermore National Laboratory. We have extended a reflectometry simulation model to accommodate $\mathrm{O}$ and $\mathrm{X}$-mode mixed polarization and
\end{abstract}


linear mode conversion between the two polarizations. A Wentzel-Kramers-BrillouinJeffreys (WKBJ) formula for linear mode conversion agrees reasonably well with direct numerical solutions of the wave equation, and we have reconstructed the magnetic pitchangle profile by matching the results of the $\mathrm{WKBJ}$ formula with the mode conversion data observed in simulations using a least-squares determination of coefficients in trial functions for the profile. The reflectometry data also yield information on fluctuations. Instrumental issues, e.g., the effects of microwave mixers and filters on model reflectometry pulses, have been examined to optimize the performance of the reflectometry diagnostics.

Class. Nos.: $52.40 \mathrm{D}, 52.70,07.60 \mathrm{H}$ 


\section{INTRODUCTION}

Reflectometry is a well-established diagnostic technique used for measuring electron density profiles and fluctuations in laboratory plasmas. ${ }^{1-7}$ Microwave sources with a range of pulse widths, $\tau_{\mathrm{p}} \sim 1-3$ ps for ultra-short pulses to $\sim 200$ ps for moderate pulses, can be used for pulsed-radar reflectometry applications. ${ }^{8-12}$ In reflectometry, a wave train of electromagnetic waves (microwaves in magnetic fusion experiments) is directed from a waveguide into a plasma. The waves propagate to a cutoff layer where they are reflected, and they transit back out of the plasma to a detector. From the accrued phase delays or from the time delays to return to the detector can be inferred some of the properties of the plasma along the propagation path of the waves. In ultra-short-pulse reflectometry a single short pulse is launched that has the desirable properties of being very broad in frequency bandwidth to span a significant spread of plasma cutoff frequencies (if the detection hardware has adequate frequency spread and resolution) and short in time compared to typical time scales on which the plasma evolves. In addition, the reflectometry diagnostic system can be cycled rapidly enough to follow the temporal evolution of the plasma. Thus, ultra-short-pulse reflectometry offers the potential of delivering both temporally and spatially resolved plasma characteristics.

This work extends our earlier computational studies of pulsed reflectometry. ${ }^{13-16}$ In our earlier work we presented the results of one-dimensional simulations of pulsed reflectometry using ordinary $(\mathrm{O})$ modes in Refs. 13 and 14, and demonstrated the robustness of density-profile reconstructions in the presence of density perturbations. In Ref. 15 we presented the results of one and two-dimensional studies of pulsed reflectometry using $\mathrm{O}$ and $\mathrm{X}$ (extraordinary) modes. Because the cutoff frequency for an $\mathrm{X}$ mode depends jointly on the electron density and the electron cyclotron frequency, the modulus of the magnetic field as a function of depth into the plasma can be deduced from the $\mathrm{X}$-mode group delays if the electron density is already known (perhaps determined by O-mode reflectometry). The use of $\mathrm{X}$ modes to reconstruct the magnetic field requires that 
the magnetic field is generally an increasing function of distance from the edge of the plasma into the interior, that accessibility condtions are satisfied, and that electron cyclotron resonances can be avoided or the associated absorption is weak. Short-pulse O-mode reconstructions of the electron density profile were found to be remarkably robust with respect to coherent density perturbations of significant amplitude (exceeding $20 \%$ of a nominal density in plasma interior) either parallel or perpendicular to the wave propagation direction. The signature of Bragg resonance in the reflectometry signals was shown to give useful information on fluctuations in Ref. 15. In Ref. 16 we presented additional imulations of pulsed reflectometry in which the reflectometry simulations were coupled to TRSICA equilibrium and transport code ${ }^{17}$ which was used to calculate experimentally .nt electron density and magnetic field profiles as input to the reflectometry mulations. A least-squares-based reconstruction algorithm for the determination of the spatial profile of the magnitude of the magnetic field also was introduced in Ref. 16.

In this paper we report progress in the theory and modeling of a set of reflectometry diagnostics proposed for use in the new Sustained Spheromak Physics Experiment (SSPX) at the Lawrence Livermore National Laboratory. SSPX proposes to use O and X-mode reflectometry to determine spatial profiles for the electron density, magnitude of the magnetic field, and the relative toroidal and poloidal components of the magnetic field. The last measurement, the magnetic pitch angle, is inferred from the linear mode conversion from $\mathrm{X}$-modes to $\mathrm{O}$-modes produced by magnetic shear along the wave propagation path through the plasma (Sec. II). We also consider some of the instrumental issues that influence the realization of the pulsed reflectometry system in the laboratory, for example, the effects of microwave mixers and filters on the reflectometry pulses and how to modify the pulses to optimize the performance of the reflectometry diagnostics (Sec. III). We conclude with a discussion of a few important physics issues that influence the success of pulsed reflectometry and some observations (Sec. IV). 


\section{LINEAR MODE CONVERSION REFLECTOMETRY}

If the scale length over which there is appreciable magnetic shear is long compared to the wavelength of the probing $\mathrm{O}$ or $\mathrm{X}$ modes, i.e., the magnetic shear is weak, the polarization vector of the probing waves rotates to follow the direction of the local magnetic field in the plasma. 18,19 However, the magnetic shear induces linear mode conversion between the $O$ and $X$ modes. ${ }^{19,20,21}$ Mixed $O$ and $X$-polarization simulations of reflectometry have been reported in Ref. 21 wherein the signature of Bragg-resonant cross-polarization scattering was identified. In the work reported in this paper, we show that the Wentzel-Kramers-Brillouin-Jeffreys (WKBJ) solution ${ }^{19,20}$ for the linear mode conversion of $\mathrm{X}$ modes to $\mathrm{O}$ modes agrees reasonably well with the direct solution of the mixed polarization wave equations in simulations and that this can be used as the basis of a reconstruction algorithm to determine the spatial profile for the magnetic pitch angle.

In our one-dimensional simulations of mixed polarization reflectometry in a cold plasma we solve the following set of equations:

$$
\begin{aligned}
& {\left[\frac{\partial^{2}}{\partial t^{2}}+\omega_{\mathrm{pe}}^{2}\right] \mathrm{E}_{\mathrm{x}}=\omega_{\mathrm{pe}}^{2}\left[\mathrm{v}_{\mathrm{z}} \mathrm{B}_{\mathrm{y}}(\mathrm{x})-\mathrm{v}_{\mathrm{y}} \mathrm{B}_{\mathrm{z}}(\mathrm{x})\right]} \\
& {\left[\frac{\partial^{2}}{\partial \mathrm{t}^{2}}-c^{2} \frac{\partial^{2}}{\partial \mathrm{x}^{2}}+\omega_{\mathrm{pe}}^{2}\right] \mathrm{E}_{\mathrm{y}}=\omega_{\mathrm{pe}}^{2} \mathrm{v}_{\mathrm{x}} \mathrm{B}_{\mathrm{z}}(\mathrm{x})} \\
& {\left[\frac{\partial^{2}}{\partial \mathrm{t}^{2}}-c^{2} \frac{\partial^{2}}{\partial \mathrm{x}^{2}}+\omega_{\mathrm{pe}}^{2}\right] \mathrm{E}_{\mathrm{z}}=-\omega_{\mathrm{pe}}^{2} \mathrm{v}_{\mathrm{x}} \mathrm{B}_{\mathrm{y}}(\mathrm{x})}
\end{aligned}
$$

for the electric fields $\left(E_{\mathrm{x}}, \mathrm{E}_{\mathrm{y}}, \mathrm{E}_{\mathrm{z}}\right)$ and

$$
\begin{gathered}
\frac{d v_{x}}{d t}=-\frac{e}{m_{e}}\left(E_{x}+v_{y} B_{z}-v_{z} B_{y}\right) \quad \frac{d v_{y}}{d t}=-\frac{e}{m_{e}}\left(E_{y}-v_{x} B_{z}+v_{z} B_{x}\right) \\
\frac{d v_{z}}{d t}=-\frac{e}{m_{e}}\left(E_{z}+v_{x} B_{y}\right)
\end{gathered}
$$

for the plasma fluid velocities $\left(v_{x}, v_{y}, v_{z}\right)$, where $B_{y}$ and $B_{z}$ are the background poloidal and toroidal magnetic field components. References 14-16 describe the numerical methods used to solve Eqs.(1-3) and some of the code validation checks. In the one-dimensional 
simulations reported here, $c=\Delta x=\Delta t=1$ defines the non-dimensional code units. As a check of the mixed polarization aspect of the numerical simulation, we undertook mixed polarization simulations of the O-mode determinations of the electron density profiles and $\mathrm{X}$-mode determinations of the magnitude of the magnetic field profiles for DIII-D tokamak and SSPX spheromak plasmas presented in Ref. 16 and obtained the same results. In the new simulations, we launched pulses that were dominantly O modes or X modes according to whether the vacuum electric field polarization of the probing waves was more nearly parallel or perpendicular, respectively, to the magnetic field at the edge of the plasma and then selected the appropriate polarization direction at the detector location.

In Ref. 20 the linear mode conversion of $\mathrm{X}$ modes to $\mathrm{O}$ modes by weak magnetic shear was demonstrated experimentally and verified with a numerical evaluation of the WKBJ solution of the linearly coupled wave equations. In that experiment the mode conversion accompanied transmission across the plasma. The excitation of the O-mode by the $\mathrm{X}$-mode in the limit of weak magnetic shear is described by 19,20

$$
\frac{\partial^{2} E_{O}}{\partial x^{2}}+k_{\|}^{2}(x) E_{O} \approx-2 \frac{d \theta}{d x} \frac{\partial E_{X}}{\partial x}
$$

where $\theta=\tan ^{-1}\left(B_{Z} / B_{y}\right)$ is the magnetic pitch angle, with approximate solution

$$
\left|\frac{E_{O}(\omega)}{E_{X}(\omega)}\right|=\left.\left|\int_{0}^{x} d x^{\prime} \frac{d \theta}{d x^{\prime}}\right| \frac{k_{X}}{k_{O}}\right|^{1 / 2} \exp \left[i \int_{0}^{x^{\prime}} d x^{\prime \prime}\left(k_{X}-k_{O}\right)\right]
$$

for single-pass mode conversion, where $E_{U}$ and $E_{X}$ are the $O$ and $X$ mode amplitudes at the frequency $\omega$, and $\mathrm{kX}_{X}$ and $\mathrm{k}_{\mathrm{O}}$ are the $\mathrm{X}$ and $\mathrm{O}$ mode wavenumbers satisfying the coldplasma linear dispersion relations for the frequency $\omega$.

Consider the case of an $\mathrm{X}$ mode propagating across the magnetic field into a model sphcromak plasma. (Alternately, the experiment could use the conversion of an initial Omode into an $\mathrm{X}$-mode to obtain the same information.) There are two branches of the Xmode dispersion relation corresponding to slow and fast modes with cutoff relations: $\omega_{1 \mathrm{c}}=$ 
$-\omega_{\mathrm{ce}} / 2+\left(\omega_{\mathrm{pe}}{ }^{2}+\omega_{\mathrm{ce}}{ }^{2 / 4}\right)^{1 / 2}$ and $\omega_{2 \mathrm{c}}=\omega_{\mathrm{ce}} / 2+\left(\omega_{\mathrm{pe}}{ }^{2}+\omega_{\mathrm{ce}} 2 / 4\right)^{1 / 2}$, respectively, where $\omega_{\mathrm{ce}}$ is the the magnitude of the electron cyclotron frequency and $\omega_{\mathrm{pe}}$ is the electron plasma frequency. In Fig. 1 we display model SSPX spatial profiles calculated in the CORSICA equilibrium and transport code for the electron cyclotron frequency and its harmonics, and the cutoff frequencies for the slow and fast $\mathrm{X}$ modes and the $\mathrm{O}$ mode. We also show the poloidal and toroidal magnetic field profiles. Figure 2 is a schematic of an incident fast X mode, (1) in Fig. 2, with frequency greater than the $\omega_{\mathrm{ce}}$ at the plasma edge and smaller than the maximum value of $\omega_{\mathrm{pe}}$ in the core of the plasma undergoing mode-conversion to an inward propagating $O$ mode until the $X$ mode encounters its cutoff, where $\omega=\omega_{2 c}$, and then it is reflected. The $\mathrm{O}$ mode continues to propagate farther into the plasma until it encounters its cutoff, where $\omega=\omega_{\mathrm{pe}}$, and then it is reflected and propagates back out of the plasma, (2) in Fig. 2. The reflected $\mathrm{X}$ mode, (3) in Fig. 2, produces an additional mode-converted $\mathrm{O}$ mode, (4) in Fig. 2, on its outward path, which $\mathrm{O}$ mode propagates directly out of the plasma and produces interference with the reflected $\mathrm{O}$ mode produced by the $\mathrm{X}$ mode on the inward path. As the two O modes, (2) and (4) in Fig. 2, produced by the mode conversion of the $\mathrm{X}$ mode on its inward and outward paths experience different path lengths, their phases differ as a function of frequency; and there is interference. In one spatial dimension the interference of the two O-mode components at the same frequency is given by

$$
\left|E_{O}(\omega)\right|=\left|E_{O}^{I}+E_{O}^{I I}\right|=\left|E_{O}^{I}(\omega)(1+\operatorname{expi} \Delta \phi)\right|, \quad \Delta \phi=2 \int_{x_{x}}^{x_{0}^{\text {cut }}} \mathrm{dx}^{\prime} k_{O}+\int_{0}^{x_{x}^{\mathrm{cut}}} \mathrm{dx}^{\prime}\left(\mathrm{k}_{\mathrm{O}}-\mathrm{k}_{\mathrm{X}}\right)
$$

where $\mathrm{kO}_{\mathrm{O}}$ and $\mathrm{kX}$ are wavenumbers respectively satisfying the $\mathrm{O}$ and $\mathrm{X}$-mode dispersion relations locally at the frequency $\omega$. The reflected $X$ and $O$ modes both acquire $-\pi / 2$ phase shifts as well on reflection at their cutoff layers which cancel one another in compuing $\Delta \phi$.

There is also single-pass mode conversion of reflected $\mathrm{X}$ modes, (7) in Fig. 2, to $\mathrm{O}$ modes, (8) in Fig. 2 , for $\omega$ greater than the maximum O-mode cutoff frequency $\omega_{\text {pe }}$ and less than the maximum $X$-mode cutoff frequency $\omega_{2 c}$. On thcir inward path the $\mathrm{X}$ modes, 
(5) in Fig. 2, mode-convert into $\mathrm{O}$ modes (6) in this frequency range that see no cutoff frequency and never return to the detector colocated with the X-mode launch point. In this case the interference does not occur.

In addition to the mode conversion of the $\mathrm{X}$ mode into the $\mathrm{O}$ mode at the same frequency and propagating in the same direction, $\mathrm{kX}_{\mathrm{X}} \rightarrow \mathrm{k}_{\mathrm{O}}$, there is mode conversion of the $\mathrm{X}$ mode into a counter-propagating $\mathrm{O}$ mode, $\mathrm{kX}_{\mathrm{X}} \rightarrow-\mathrm{k}_{\mathrm{O}}$. There is mode conversion in the inward and outward paths of the X mode as before, and there is single-pass mode conversion on the $\mathrm{X}$-mode inward path for frequencies above the maximum plasma frequency. The expression for the single-pass mode conversion for $\mathrm{k}_{X} \rightarrow-\mathrm{k}_{\mathrm{O}}$ is the same as in Eq.(4) with $\mathrm{k}_{\mathrm{O}} \rightarrow-\mathrm{k}_{\mathrm{O}}$,which causes increased phase mixing in the integration and greatly reduces the resulting mode-conversion amplitude. There is also a small amount of mode conversion of the mode-converted $\mathrm{O}$ mode back to the $\mathrm{X}$ mode, which we have neglected in our WKBJ analysis.

In Fig. 3 we show the results of a direct numerical solution of the one-dimensional wave and plasma equations, Eqs.(1) and (2), for mixed $\mathrm{X}$ and $\mathrm{O}$ polarizations and the electron density and magnetic field profiles obtained from the CORSICA code displayed in Fig. 1. The time histories of the reflected $\mathrm{X}$ and $\mathrm{O}$ modes resulting from injecting an $\mathrm{X}$ mode with peak pulse amplitude equal to unity are shown. In Fig. 4 we present a comparison of the results of numerically integrating Eqs.(4) and (5) using the true electron density and magnetic field spatial profiles to the observed values of $\left|E_{O}(\omega) / E_{X}(\omega)\right|$ in the direct solution of the wave and plasma equations. Equations (4) and (5) were integrated using Simpson's rule. The Fourier amplitudes $E_{O}(\omega)$ and $E_{X}(\omega)$ were computed as a simulation diagnostic by performing a fast Fourier transform on the time histories and locally averaging these amplitudes over a frequency width $\Delta \omega / \omega \sim 10^{-2}$. This averaging smoothed fine scale structure in the data. The results of Eqs.(4) and (5) were locally averaged in frequency in likewise fashion to make the comparison shown in Fig. 4. We note that $\left|\mathrm{E}_{\mathrm{O}}(\omega) / \mathrm{E}_{\mathrm{X}}(\omega)\right| \leq 15-20 \%$ over the range of frequencies so that the energy 
attenuation due to the mode conversion was very small, which allowed us to use the measurement of the reflected $\left|\mathrm{E}_{X}(\omega)\right|$ in computing $\left|\mathrm{E}_{\mathrm{O}}(\omega) / \mathrm{E}_{\mathrm{X}}(\omega)\right|$.

We found that in the frequency range where there was double-pass mode conversion, i.e., frequencies below the maximum plasma frequency, a better fit of the WKBJ-predicted mode conversion amplitudes was obtained by increasing the phase calculated in Eq.(5) by 5\%, i.e., $\phi \rightarrow 1.05 \phi$. The results shown in Fig. 4 contain this correction factor. This correction was an empirically determined compensation for the breakdown of WKBJ for wave packets with turning points approaching the top of a hill. By comparing WKBJ theory to the direct numerical solution of the wave equation for the cutoff profiles in Fig. 1 or to the exact analytical solution for a parabolic potential in terms of parabolic cylinder functions, we concluded that the WKBJ effective wavenumber and the group velocity were too small for turning points approaching the top of a hill with result that the accumulated phase $\phi$ in Eq.(5) was too small and the corresponding group delays too long without some correction. Without the phase correction factor, the WKBJ result captured the behavior of $1 \mathrm{E}_{\mathrm{O}}(\omega) / \mathrm{E}_{X}(\omega) \mid$ as a function of frequency fairly well, but the relative spacing of the local minima and maxima in the curve was a little too wide. Fairly good agreement for the WKBJ predictions of the mode conversion was obtained with the small correction to the phase.

If the WKBJ prediction of the mode conversion using the true electron density and magnetic field profiles is reliable then minimizing the observed differences between the values of $\left|\mathrm{E}_{O}(\omega) / \mathrm{Ex}_{X}(\omega)\right|$ given by Eqs.(4-5) and those obtained by direct measurement for a set of frequencies can be the basis for determining unknown coefficients in a test profile for the magnetic pitch angle $\theta$. 'l'his assumes that we already have a knowledge of the electron density and modulus of the magnetic field profiles with which to compute $\mathrm{k}_{\mathrm{X}}$ and $\mathrm{k}_{\mathrm{O}}$ locally in space. We suggest that the electron density and mod-B spatial profiles can be obtained from the group delay data for $\mathrm{O}$ and $\mathrm{X}$-mode pulsed reflectometry, and that the mode-conversion data can be derived from reflectomeiry amplitude measurements. We 
found it advantageous in the pitch-angle reconstruction algorithm to include explicitly as many constraints as possible in the pitch-angle trial functions and to minimize the number of unknown coefficients to be determined. For applications to the spheromak, we assume that the reflectometry ray paths lie on the plasma midplane and, based on the expected plasma equilibria calculated in CORSICA, that the magnetic field is almost entirely poloidal at the edge of the plasma current layer and is entirely toroidal on the magnetic axis. A convenient trial function for the magnetic pitch angle constrained to give $\theta=0$ at the plasma edge and $\theta=\pi / 2$ at the magnetic axis is given by $\theta=(\pi / 2)\left(\mathrm{a}_{1} \mathrm{X}^{1.5}+\mathrm{a}_{2} \mathrm{X}^{2.5}+\left(1 .-\mathrm{a}_{1}-\mathrm{a}_{2}\right) \mathrm{X}^{3.5}\right)$ with $X=\left(x-a_{3}\right) /\left(x_{a_{x i s}}-a_{3}\right)$ and unknown coefficients $\left\{a_{1}, a_{2}, a_{3}\right\}$. Note that $a_{3}$ is not necessarily equal to the edge of the plasma. Figures 5 and 6 display results for the leastsquares reconstruction of the magnetic pitch angle for two reflectometry simulations using the SSPX model equilibrium depicted in Fig. 1. There were 22 frequency channels for which there were values of $I \mathrm{E}_{\mathrm{O}}(\omega) / \mathrm{E}_{X}(\omega) \mid$. The actual and reconstructed profiles for $\theta$ and $\mathrm{d} \theta / \mathrm{dx}$ as well as the values of $\left|\mathrm{E}_{\mathrm{O}}(\omega) / \mathrm{E}_{X}(\omega)\right|$ observed in the simulations and deduced from the reconstructed profiles are displayed in Figs. 5 and 6. In Fig. 6 a magnetic shear perturbation was superposed on the equilibrium magnetic profiles corresponding to a peak amplitude perturbation in the toroidal magnetic field equal to 0.02 of the magnitude of the poloidal magnetic field at the plasma edge. The trial function for $\theta(\mathrm{x})$ was augmented to include a perturbation of the form $\delta \theta(x)=a_{4} \cos \left[a_{7}\left(x-a_{5}\right)\right] \exp \left[-\left(x-a_{5}\right)^{2} / a_{6}{ }^{2}\right]$, where $\left\{\mathrm{a}_{4}, \mathrm{a}_{5}, \mathrm{a}_{6}, \mathrm{a}_{7}\right\}$ are additional unknown coefficients to be determined by the least-squares routine which govern the amplitude, wavenumber, center position, and localization width of the perturbation. We constrained $a_{4}$ to give a small-amplitude perturbation, as to lie inside the plasma, $a_{6}$ to produce a localized perturbation relative to the plasma radius, and a7 to lie in a range of physically acceptable wavenumbers.

The reconstructions of $\theta, d \theta / d x$, and $\left|E_{O}(\omega) / E_{X}(\omega)\right|$ shown in Figs. 5 and 6 are reasonably good. The magnitude of the magnetic field and the magnetic pitch angle determine the toroidal and poloidal vector components of the magnetic field from whose 
radial derivatives in the midplane can be calculated the curl of the magnetic field and, hence, the current profile. In laboratory applications it will be necessary to determine the $\mathrm{O}$ and $\mathrm{X}$ mode polarization directions to a degree of angular precision such that the projection of the actual X-mode electric field vector onto the supposed O-mode polarization direction is much smaller than the mode-converted O-mode amplitude.

\section{MODELING REFLECTOMETRY PULSE CHARACTERISTICS}

The ultra-short-pulse reflectrometer being constructed for SSPX will incorporate up to 56 frequency channels, ranging from frequencies characteristic of those expected for the core plasma, $>140 \mathrm{GHz}$, to those expected for the edge, $\sim 35 \mathrm{GHz} .{ }^{22}$ The device consists of transmitter and receiver sections, coupled by antennas (horns) to the vacuum vessel and the plasma. Because the evaluation of plasma parameters depends on the measurement of the group delay in cach channcl, it is csscntial that dispersion or other distortion in the electronics be small enough that an accurate measurement can be made. Furthermore, analysis will be required to correct measured signals for instrumental effects when the actual experiment begins.

The implementation of the pulsed radar for SSPX uses a chirped pulse, as can be seen from the block diagram of the system shown in Fig. 7, which includes qualitative examples of the signals at each stage. A short pulse (roughly Gaussian in time) is converted into a dispersed frequency (6.5-18 $\mathrm{GH}$.) by transmission through a waveguide close to cutoff. This signal, following amplification, is used in all the high frequency channels by mixing with one of several high-frequency, cw waves, generated by a Gunn diode. The result is a dispersed signal which is launched into the plasma as shown. The reflected signal is then down-converted and split into 8 sub-channels by band-pass filters approximately $0.1 \mathrm{GHz}$ wide, and detected by an amplitude level-detector following a low-

pass filter. (The full set of frequency channels is obtained by switching (at $400 \mathrm{kHz}$ ) between 7 high-frequency signals and/or polarizations.) The group delay time for each 
frequency band is determined by changes in the time difference between the detected return signal and a "start-time" determined from the initial short pulse.

Analysis of the microwave system treats each stage of the transmitter and receiver as a linear filter. The plasma reflector is also treated as a filter for the present, although the analysis will later be coupled to the simulation model discussed in this paper. An example calculated by the model, including characteristics of actual microwave components, is shown in Fig. 8. The time between signals can be determined from the differences between the first signals $(\sim 1.5 \mathrm{~ns})$, the delays at half amplitude $(1.60 \mathrm{~ns})$ or the pulse peaks (1.67 ns). These correspond closely to the predicted time (1.53 ns); the delays at half amplitude yield the reflection to better than $1 \mathrm{~cm}$. In the actual device, calibrations of the time delays can eliminate even this error. However, in the actual measurement effects such as noise, the multi-dimensional nature of the plasma, and inaccuracies in the model are likely to increase this error significantly. It is anticipated that as the reflectometry system becomes operational, the combination of actual data and this transmitter model coupled to the CORSICA analysis will play a major role in interpreting the measurement.

\section{DISCUSSION}

There are several physics issues that influence the viability of using pulsed reflectometry to make measurements of density and magnetic field spatial profiles. Although it is beyond the scope of this paper to treat these issues in any detail, we will comment on the effects on reflectometry of microwave beam spreading due to diffraction, the inadequacy of the WKBJ prescription for group delays in spatial cutoff profiles near a local maximum, and density and magnetic field fluctuations.

In laboratory applications of reflectometry the spreading of the probing microwaves due to diffraction will affect the amplitude of the reflected waves at the detector. The group delay measurements used for profile reconstructions demand sufficient microwave amplitudes at the detectors to differentiate signals from noise and to identify the peak of the 
pulse in a frequency channel unambiguously. The group delay measurement does not require a precise quantitative measurement of the pulse amplitude. However, the ratio of the mode-converted O-mode amplitude to the $\mathrm{X}$-mode amplitude in a frequency channel involves the measurements of both the $\mathrm{O}$ and $\mathrm{X}$-mode amplitudes. These amplitudes are affected by the spreading of the wave packets in the plasma. We have made an analytical calculation of the beam spreading of freely propagating, $\mathrm{O}$ and $\mathrm{X}$ modes, as well as modeconverted $\mathrm{O}$ modes driven by a spreading $\mathrm{X}$-mode beam that is cylindrically symmetric with respect to the beam axis. The analysis makes use of an eikonal expansion for the propagation parallel to the incident propapagation direction and a paraxial approximation. ${ }^{23}$ As the beam spreads in propagating away from a focus, the amplitude on axis decreases to conserve energy flux. The fast $\mathrm{X}$ mode spreads faster than does the $\mathrm{O}$ mode at the same frequency because the wave frequency is farther above the O-mode cutoff, which results in the O-mode group velocity being faster than that of the $\mathrm{X}$ mode yielding a smaller O-mode group delay. The mode-converted O-mode inherits the beam width of the $\mathrm{X}$ mode that drives it. When the mode-converted $\mathrm{O}$ mode propagates beyond the $\mathrm{X}$-mode cutoff, its beam width spreads at the slower rate of freely propagating $\mathrm{O}$ modes. We conclude that for cylindrically symmetric $\mathrm{X}$ and $\mathrm{O}$ modes the ratio of the mode-converted O-mode Fourier amplitude in a specific frequency channel to the $\mathrm{X}$ mode amplitude at the end of a single pass on the beam axis will be given by Eq.(4) with a modification in the first integral on the right side to include a new factor equal to the axial beam attenuation of the $\mathrm{X}$-mode amplitude due to spreading as a function of $\mathrm{x}^{\prime}$ divided by the total $\mathrm{X}$-mode attenuation over the entire pass, which factor is clearly $\geq 1$. The double-pass mode conversion and interference will be altered by the additional path length and resulting beam spreading and axial attenuation of the $\mathrm{O}$ modes produced on the inward transit that must propagate farther into the plasma before reflecting and then superposing with the $\mathrm{O}$ modes produced by the $\mathrm{X}$ modes on their outward pass (as diagrammed in Fig. 2). 
The reflectometry reconstruction algorithms for electron density, magnetic field modulus, and magnetic pitch angle profiles rely on WKBJ formulae. However, WKBJ theory is formally invalid near the wave cutoff. This is not a difficulty for cutoff profiles that are locally linear in space near the cutoff, in which case the asymptotic theory for the Airy function indicates that the WKBJ phase and group delay are very good approximations provided that $\omega \mathrm{L} / \mathrm{c}>>1$, where $\mathrm{L}$ is the local scale length of the dielectric function near cutoff. ${ }^{24}$ However, as the wave frequency approaches a local maximum or plateau in a cutoff profile with negative curvature in space, the WKBJ phase delay and group velocity are smaller than their true values; and the WKBJ group delay is logarithmically divergent. By doing reconstructions from the experimentally determined group delays (or the simulation values calculated from a full-wave solution) that are smaller than the WKBJ group delays would be for the same profile, one infers a cutoff profile from WKBJ theory that is steeper than the actual profile. We have observed this in O-mode electron density reconstructions (Fig. 1 in Ref. 15) and in X-mode mod-B reconstructions (Fig. 5 in Ref. 16). This can be remedied with a semi-empirical reduction of the WKBJcalculated group delays using the numerical solution of the full-wave equation for arbitrary cutoff profiles (as in the mode conversion algorithm's calculation of phase delays in Sec. II) or analytically if the cutoff profile near a local maximum is parabolic in shape to good approximation. The parabolic profile is exactly solvable in terms of parabolic cylinder functions 25 and yields group delays that are finite for all frequencies up to and including the top of the cutoff profile. By appropriately reducing the logarithmically divergent WKBJ group delays as the wave frequencies approach a local maximum or plateau in the cutofffrequency profile, we have been able to significantly improve the X-mode cutoff-profile reconstructions in SSPX reflectometry simulations.

Reflectometry is also used for fluctuation measurements. ${ }^{5-7,15,21}$ Mazzucato ${ }^{26}$ has demonstrated in a numerical calculation that random poloidal density fluctuations can render phase-delay measurements unreliable in continuous wave, frequency-modulated 
reflectometry at relatively small fluctuation amplitudes (a few percent relative amplitude). In contrast, we have found that direct measurements of the group delay (determined by discriminating for the passing peak of the pulse in each frequency channel) and the resulting profile reconstructions were robust with respect to relatively large spatially coherent radial or poloidal, density or magnetic field ripples (tens of percent relative amplitude in the cutoff profiles) in our one and two-dimensional simulations of pulsed reflectometry in Refs. 13-16. Mazzucato's calculation assumed a monochromatic incident plane wave and investigated the continuous wave response and interference in two dimensions. His calculation did not address short pulses launched from a localized spatial source (a waveguide in the experiments). In pulsed reflectometry with a localized source, microwaves traveling off the beam axis transit a longer path in returning to the detector, which results in increased attenuation because of the beam spreading effects and a longer time delay compared to the axially propagating microwaves that dominate the group delay measurements. Thus, the interference effects on the direct group delay measurements in short-pulse reflectometry are very different and evidently much less deleterious than in the scenario calculated in Ref. 26.

There is a specific aspect of the group delays observed in pulsed reflectometry that may be especially relevant in monitoring plasma behavior in SSPX. Magnetohydrodynamic turbulence is significant in typical spheromak plasmas. The development of a finite-sized magnetic island can be accompanied by a flattening of the radial density profile in the island. When a plateau forms in the $\mathrm{O}$ or $\mathrm{X}$-mode cutoff profile, the group delays for frequencies approaching the plateau cutoff frequency become increasingly large. There follows a sharp decrease in the group delays for higher wave frequencies for which the cutoff profile is again increasing with increasing distance into the plasma, and the group delays finally begin to increase with increasing frequency farther into the plasma if the cutoff profile continues to rise (see Fig. 1 of Ref. 15). The swelling, 
contraction, and radial motion of the island can be tracked in time by following the changes in the inverted group delay pattern over a series of reflectometry pulses. 27

The proposed use of pulsed $\mathrm{O}$ and X-mode reflectometry in the SSPX experiment offers tests of exciting new diagnostic capability. In the future we intend to augment the calculations presented here with two-dimensional mixed polarization simulations of pulsed reflectometry to provide additional theory and modeling support for the SSPX experiment.

\section{ACKNOWLEDGMENTS}

We are grateful to D. N. IIill, T. B. Kaiser, L. L. LoDestro, N. C. Luhmann, Jr., L. D. Pcarlstein, D. D. Ryutov, and E. A. Williams for their technical input and interest in this research. This work was supported by the U.S. Department of Encrgy under Contract No. W-7405-ENG48 at the Lawrence Livermore National Laboratory.

\section{References}

1J.L. Doane, E. Mazzucato, and G.L. Schmidt, Rev. Sci. Instrum. 52, 12 (1981); F. Simonet, Rev. Sci. Instrum. 56, 664 (1985)

${ }^{2}$ A.C.C. Sips and G.J. Kramer, Plasma Phys. Contr. Fusion 35, Plasma Phys. Control. Fusion 35, 743 (1993).

${ }^{3}$ K.W. Kim, E.J. Doyle, W.A. Peebles, A. Ejiri, N.C. Luhmann, Jr., and C.L. Rettig, Rev. Sci. Instrum. 66, 1229 (1995); and references therein.

${ }^{4}$ I.H. Hutchinson, Principles of Plasma Diagnostics (Cambridge: Cambridge University Press), 1987.

5I.H. Hutchinson, Plasma Phys. Control. Fusion 34, 1225 (1992); E. Mazzucato and R. Nazikian, Plasma Phys. Control. Fusion 33, 261 (1991); and references therein.

6N.Bretz, Phys. Fluids B 4, 2414 (1992).

${ }^{7}$ C. Laviron, A.J. H. Donne, M.E. Manso, and J. Sanchez, Plasma Phys. Control. 
Fusion 38, 905 (1996).

${ }^{8}$ C.W. Domier, E. Chung, E.J. Doyle, H.-X.L. Liu, A. Lapidus, N.C. Luhmann, Jr., W.A. Peebles, X.-H. Qin, T.L. Rhodes, and L. Sjogren, Rev. Sci. Instum. 6 3, 4666 (1992); C.W. Domier, N.C. Luhmann, Jr., A.E. Chou, W.-M. Zhang, and A.J. Romanowsky, Rev. Sci. Instum. 65, 399 (1995).

${ }^{9}$ V.F. Shevchenko, A.A. Petrov, and V.G. Petrov, International Jrnl of Infrared and Millimeter Waves 14, 1755 (1993).

${ }^{10}$ N.C. Luhmann, Jr. et al., Diagnostics for Contemporary Fusion Experiments (Societa Italiana di Fisica, Varenna, Italy, 1991), pp. 135-178; B. Branas, T. Estrada, P. de la Luna, and A.P. Navarro, Diagnostics for Contemporary Fusion Experiments (Societa Italiana di Fisica, Varenna, Italy, 1991), pp. 739746.

11 V.A. Vershkov and V.A. Zhuravlev, Sov. Phys, Sov. Phys. Tech. Phys. 32, 523 (1987).

${ }^{12}$ C.A.J. Hugenholtz and S. H. Heijnen, Rev. Sci. Instum. 62, 1100 (1991).

${ }^{13}$ B.I. Cohen, B.B. Afeyan, A.E. Chou, and N.C. Luhmann, Jr., Rev. Sci. Instrum. 66, 1241 (1995).

${ }^{14}$ B.I. Cohen, B.B. Afeyan, A.E. Chou, and N.C. Luhmann, Jr., Plasma Phys. Control. Fusion 37, 329 (1995).

15B.I. Cohen, T.B. Kaiser, and J.C. Garrison, Rev. Sci. Instrum. 68, 1238 (1997).

16B.I. Cohen, L.L. LoDestro, E.B. Hooper, and T.A. Casper, Plasma Phys. Control. Fusion 40, 75 (1998).

${ }^{17}$ L.L. LoDestro and L.D. Pearlstein, Phys. Plasmas 1, 90 (1994); A. Tarditi, R.H. Cohen, J.A. Crotinger, G.D. Porter, T.D. Rognlien, A.I. Shestakov, and G.R. Smith, Contrib. Plasma Phys. 36, 132 (1996); T.A. Casper, J. Crotinger, W. Meyer, J. Moller, L.D. Pearlstein, B.W. Rice, B.W. Stallard, L.L. Lao, and T.S. Taylor, "Modeling of Current Profile Evolution and Equilibria in Negative Central Shear Discharges in the DIII- 
D Experiment," 23rd European Physical Society Conference on Controlled Fusion and Plasma Physics, Kiev, Ukraine, 24-28 June, 1996, Volume 20C, Part I, p. 295. 18M.A. Heald, Plasma Physics 6, 617(1964).

${ }^{19}$ I. Fidone and G. Granata, Nucl. Fusion 11, 133 (1971).

20R. Cano, M.J. Schwartz, and B. Zanfagna, Phys. Fluids 15, 479 (1972).

${ }^{21}$ N. Katsuragawa, H. Hojo, and A. Mase, "Simulation Study on Cross Polarization Scattering of Ultra-short-pulse Electromagnetic Waves," Proc. Int. Conf. on Plasma Physics (Nagoya, 1996) p. 1738; National Institute for Fusion Science Research Report NIFS-462 (Nov. 1996), submitted to J. Phys. Soc. Japan.

${ }^{22}$ Y. Roh, C.W. Domier, N.C. Luhmann, Jr., B.I. Cohen, E.B. Hooper, and M. C. Spang, "Ultrashort Pulse Reflectometry (USPR) Diagnostic for Density and Magnetic Field Profiles Determination on SSPX," in Proceedings of the 12th Topical Conference on High-Temperature Plasma Diagnostics, Princeton, NJ (June 7-11, 1998).

${ }^{23}$ B.I. Cohen, B.F. Lasinski, A.B. Langdon, and E.A. Williams, Phys. Plasmas 4, 956 (1997).

${ }^{24} \mathrm{M}$. Abramowitz and I.A. Stegun, Handbook of Mathematical Functions, (Dover Publications, New York, 1965), 10.4.79.

25M. Abramowitz and I.A. Stegun, op. cit., 19.16 and 19.24 .

26E. Mazzucato, Rev. Sci. Instum. 69, 1691 (1998).

27P.C. De Vries, A.J.H. Donne, S.H. Heijnen, C.A.J. Hugenholtz, A. Kramer-Flecken, F.C. Schuler, and G. Waidman, Nucl. Fusion 37, 1641 (1997). 


\section{FIGURE CAPTIONS}

Figure 1. (a) SSPX model equilibrium profiles calculated in CORSICA showing the cutoff frequencies and electron cyclotron harmonic frequencies (in dimensionless code units) as functions of distance in the plasma midplane.(b) Toroidal and poloidal magnetic fields in dimensionless code units $\left(\omega_{\mathrm{ce}, \mathrm{z}}\right.$ and $\left.\omega_{\mathrm{ce}, \mathrm{y}}\right)$.

Figure 2. Diagram of single-pass and double-pass mode conversion of $\mathrm{X}$ to $\mathrm{O}$ modes.

Figure 3. Time histories of the reflected (a) X modes and (b) mode-converted $\mathrm{O}$ modes in a one-dimensional reflectometry simulation for the model SSPX equilibrium shown in Fig. 1.

Figure 4. Comparison of the simulation observations and the WKBJ calculations of the ratio $\left|E_{O}(\omega) / E_{X}(\omega)\right|$ of the mode-converted amplitude to the incident wave amplitude as a function of frequency using the true equilibrium profiles shown in Fig. 1.

Figure 5. Comparison of the simulation observations and the WKBJ-based least-squares reconstructions of (a) the ratio $\left|\mathrm{E}_{\mathrm{O}}(\omega) / \mathrm{EX}_{\mathrm{X}}(\omega)\right|$ of the mode-converted amplitude to the incident wave amplitude as a function of frequency $(\mathrm{rad} / \mathrm{sec})$, (b) the magnetic pitch angle $\theta$ vs. $x(\mathrm{~cm})$, and $(\mathrm{c}) \mathrm{d} \theta / \mathrm{dx}(\mathrm{rad} / \mathrm{cm})$ vs. $\mathrm{x}(\mathrm{cm})$. The range of frequencies in Fig. 5 is a subset of the range in Fig. 4.

Figure 6. Comparison of the simulation observations and the WKBJ-based least-squares reconstructions of (a) the ratio $\left|\mathrm{E}_{\mathrm{O}}(\omega) / \mathrm{EX}_{\mathrm{X}}(\omega)\right|$ of the mode-converted amplitude to the incident wave amplitude as a function of frequency ( $\mathrm{rad} / \mathrm{sec})$, (b) the magnetic pitch angle $\theta$ vs. $x(\mathrm{~cm})$, and (c) $d \theta / \mathrm{dx}(\mathrm{rad} / \mathrm{cm})$ vs. $x(\mathrm{~cm})$, with a magnetic-shear perturbation included $\left(\delta \mathrm{B}_{Z} / \mathrm{B}_{0}=0.02\right)$, where $\mathrm{B}_{0}$ is the magnetic-field strength at the edge of the plasma.

Figure 7. Block diagram for an ultra-short-pulse reflectometry system using chirped pulses. The chirped signal $(7-18 \mathrm{GHz})$ is mixed with each of $7 \mathrm{cw}$ signals generated by Gunn oscillators; shown is the $32 \mathrm{GHz}$ channel. Following reflection from the plasma, bandpass filters sample different frequencies, in the $39-50 \mathrm{GHz}$ range for this example, to determine the time delay for the round trip to the reflection point in the plasma. 
Figure 8. Comparison of the "start signal" and signal detected after reflection for O-mode measurement of the density. The start signal timing mark is generated by square-law, lowpass detection of the signal following the dispersive $(6.5-18 \mathrm{Ghz})$ waveguide. The Omode wave is reflected from a parabolic density profile with peak density $1.0 \times 10^{20} \mathrm{~m}^{-3}$ and half-width $0.2 \mathrm{~m}$. The reflected signal is filtered with a bandwidth of $\pm 0.1 \mathrm{Ghz}$ about 65.7 Ghz, followed by a square-law, low-pass filter. 
(a)

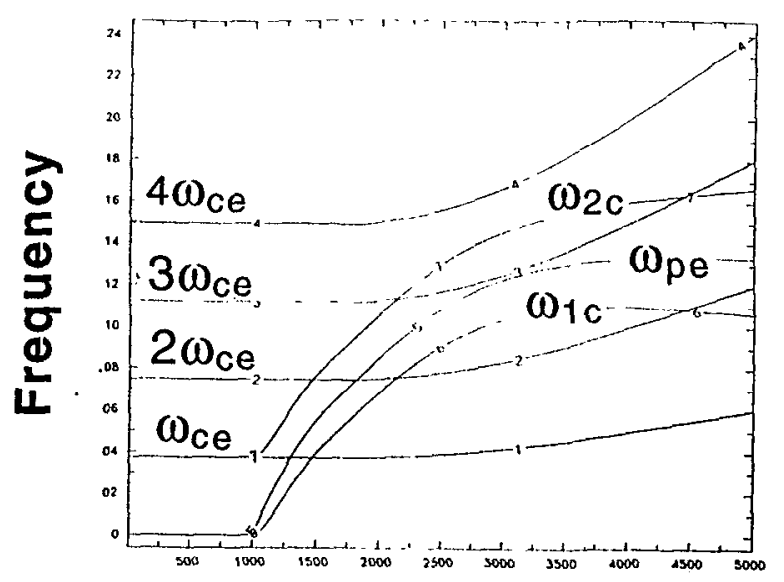

(b)

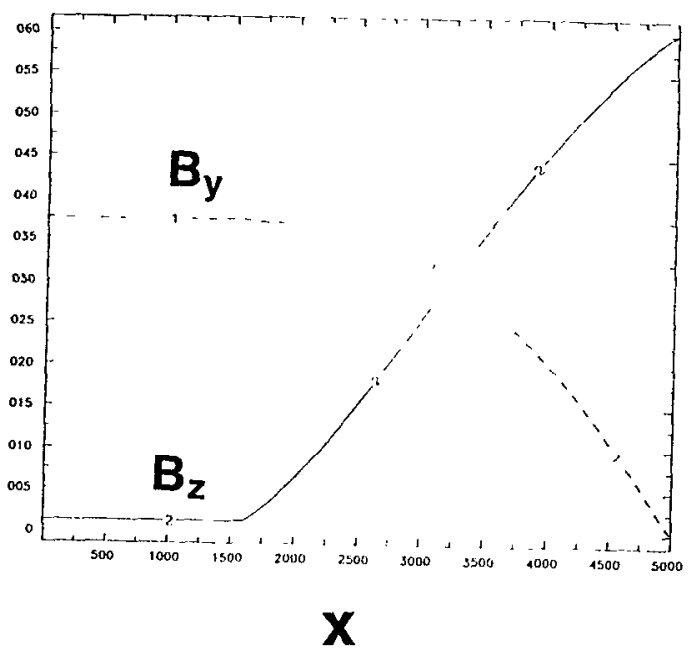

Figure 1 


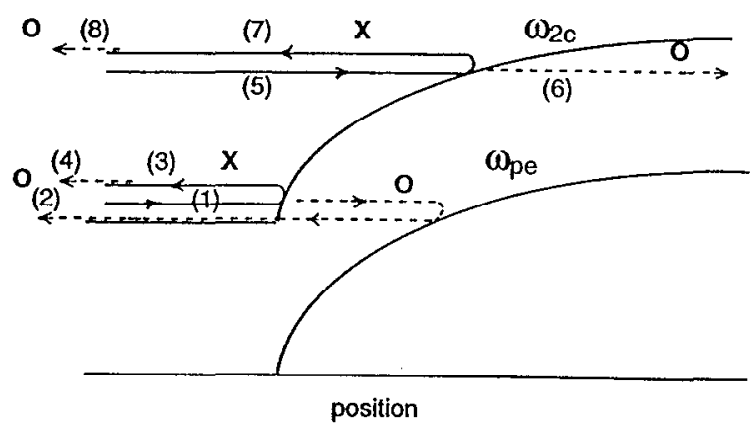

Figure 2 


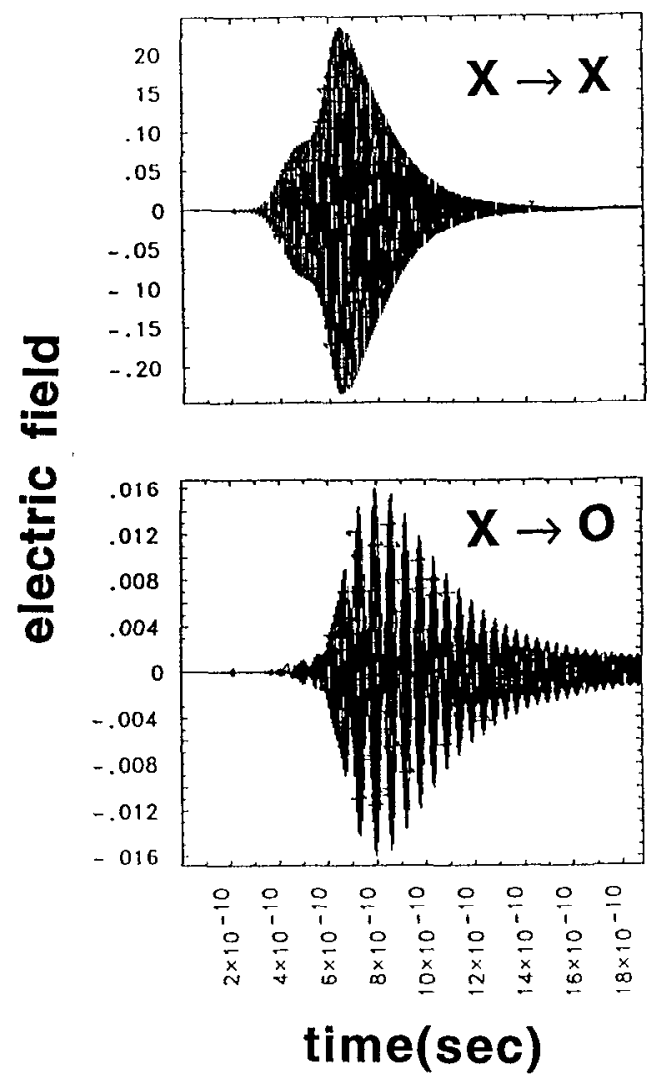

Figure 3 


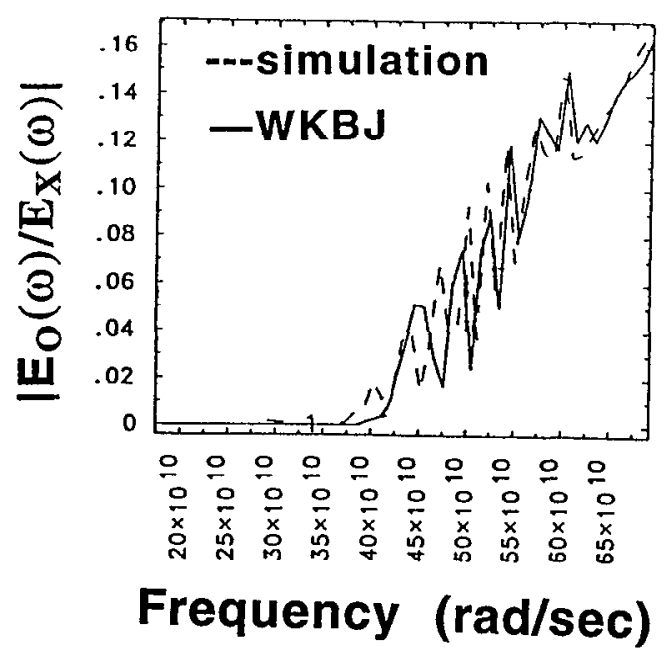

Figure 4 


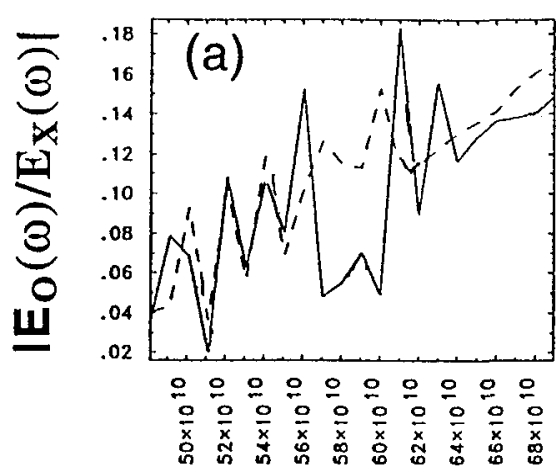

Frequency ( $\mathrm{rad} / \mathrm{sec})$
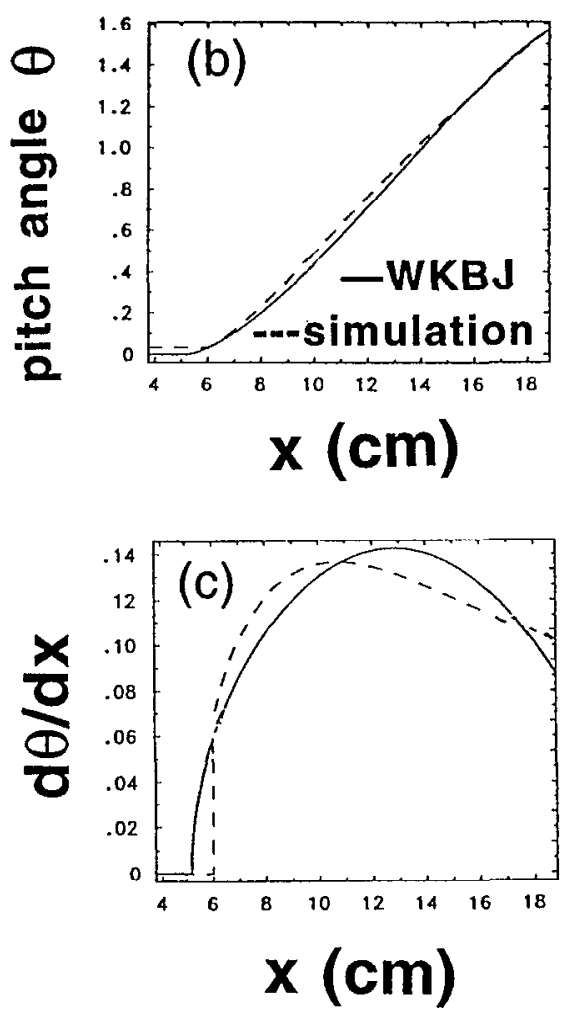

Figure 5 


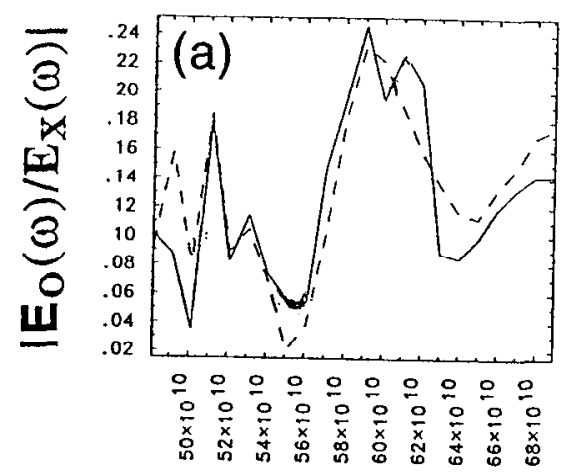

Frequency ( $\mathrm{rad} / \mathrm{sec})$
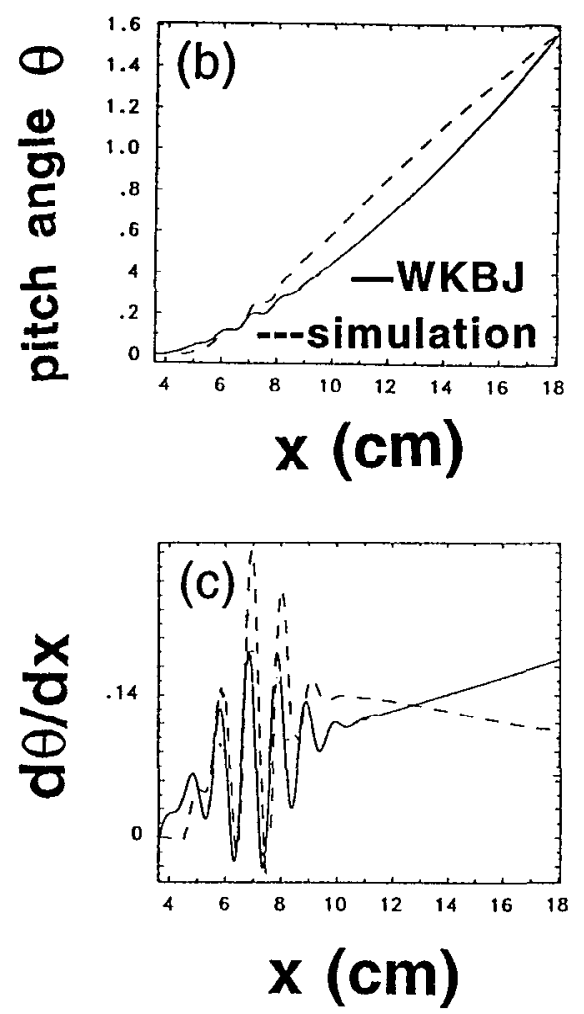

Figure 6 
Impulse Generator Signal

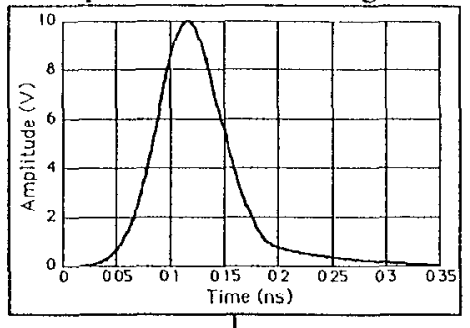

1
Signal from Waveguide

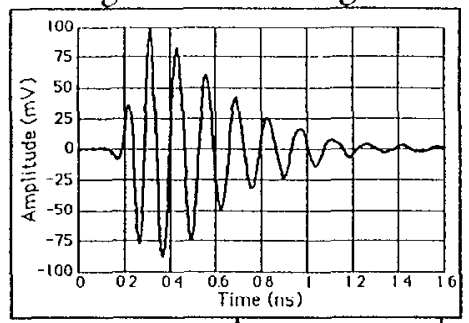

Frequency Range.

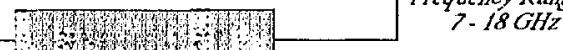

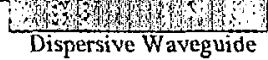

Post-Detector Signal

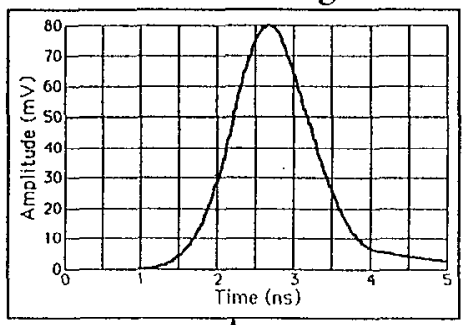

4
Post-Bandpass Filter Signal

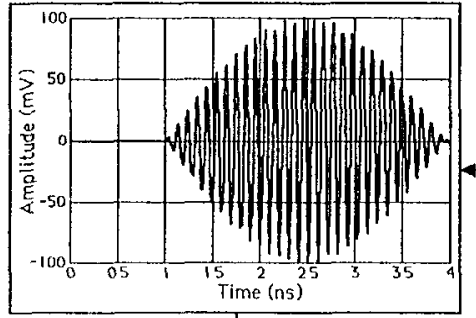

Fruguency Range. $10.4+0.1 \mathrm{GIIZ}$

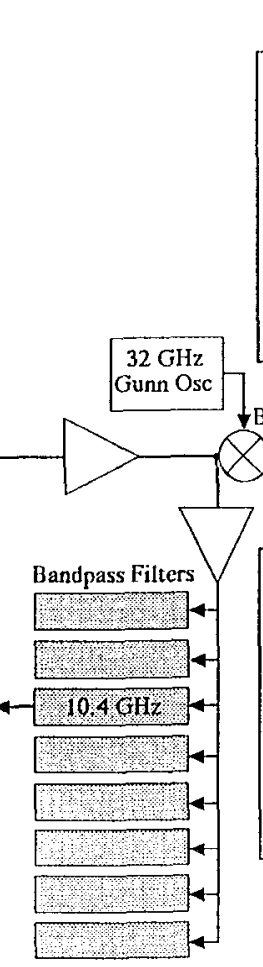

Launched Signal

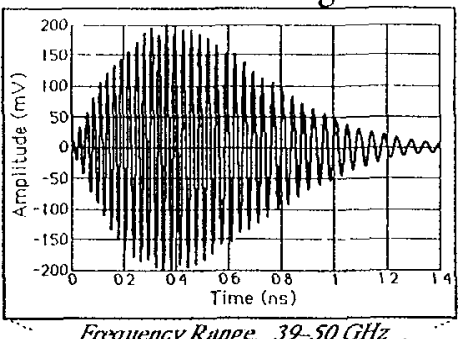

Frequency Range. $3950 \mathrm{GHz}$

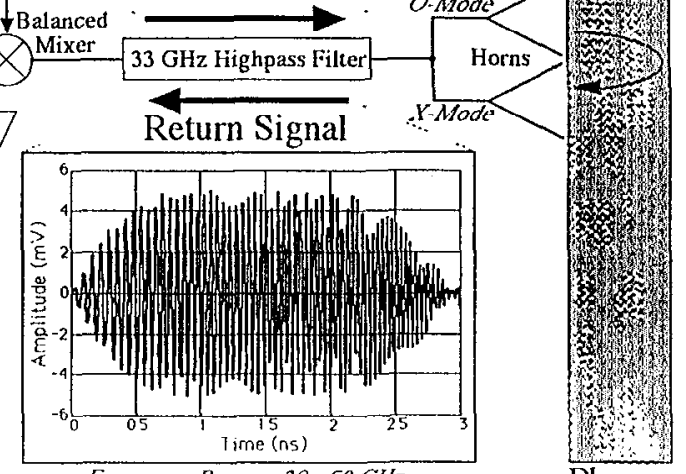

Frtyuency Ranges. $39-50 \mathrm{GHz}$

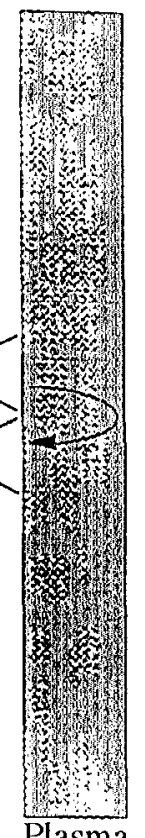

Plasma

Figure 7 


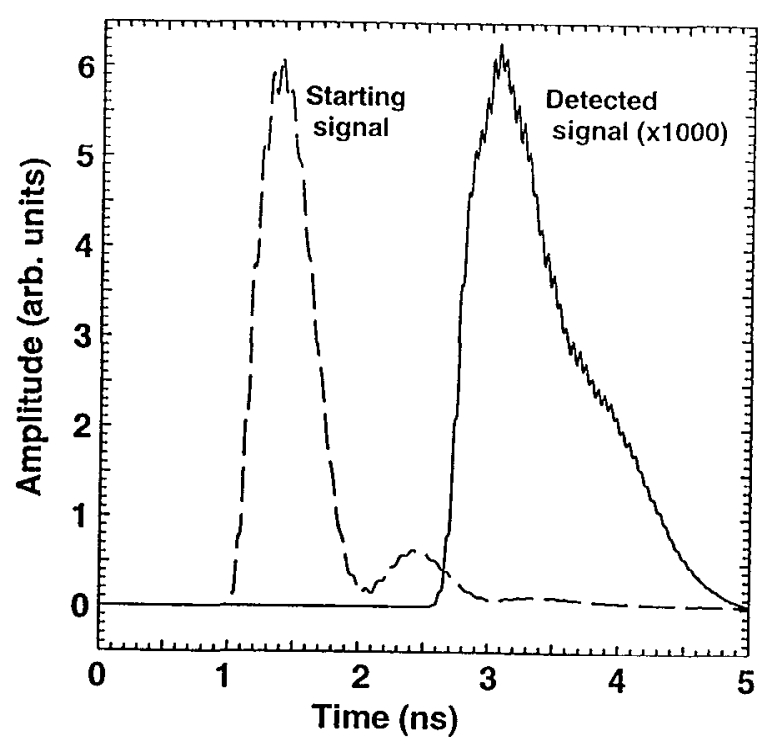

Figure 8 


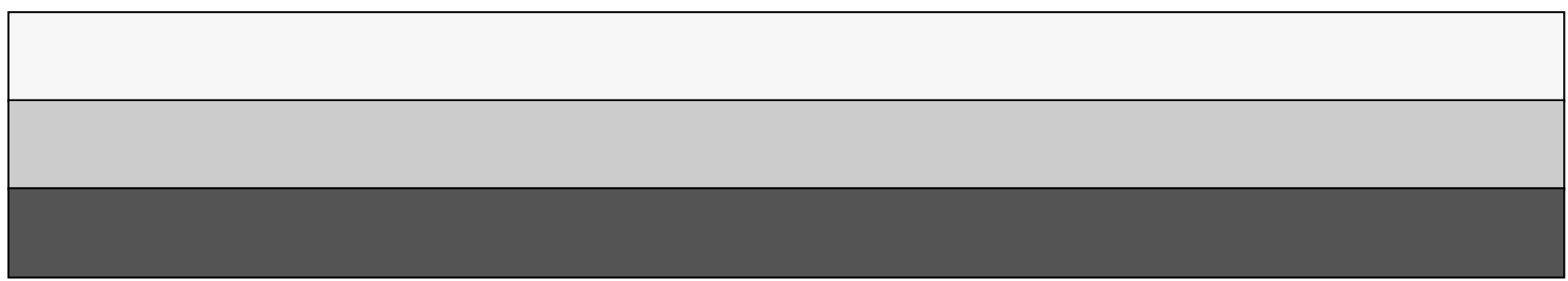

